\title{
Spatial and contextual factors in beginning reading: Evidence for PSG-CSG complements to developing automaticity?
}

\author{
DENNIS F. FISHER \\ Behavioral Research Directorate, Human Engineering Laboratory, \\ Aberdeen Proving Ground, Maryland 21005 \\ and
}

\author{
WILLIAM E. MONTANARY \\ Loyola College, Baltimore, Maryland 21210, and Behavioral Research Directorate, Human Engineering \\ Laboratory, Aberdeen Proving Ground, Maryland 21005
}

\begin{abstract}
Two experiments were designed to examine the development of spatial and contextual sensitivity during early reading. Second and fifth graders read second-and fifth-grade level paragraphs that were either normally typed or had the spaces between the words filled. Both groups read the second-grade paragraphs and the normally typed paragraphs fastest, while the filled fifth-grade paragraphs were read slowest. The data were interpreted as providing evidence for a skill acquisition hierarchy that attempts to resolve contradictions previously reported by Hochberg (1970), while providing a good complement to LaBerge and Samuel's (1974) notions of automaticity.
\end{abstract}

Hochberg (1970) has described an experiment (Hochberg, Levin, \& Frail, Note 1) in which oral reading efficiency was examined in fast- and slow-reading second and fiftil graders. These children read secondgrade level paragraphs that were normally typed and paragraphs that had the spaces between the words filled with symbols. All groups were found to have reduced oral reading fluency when word-boundary cues were minimized by filling the spaces; however, the greatest decrements occurred for the older slow-reading and younger fast-reading children.

Hochberg (1970) argued that, with experience, reading progresses from a letter-by-letter or word-byword analysis to one which incorporates larger units of information, such as a number of words or a phrase. His data were interpreted as indicating that the younger children were reading word by word without the benefit of peripheral retinal cues, and that adding symbols between the words only led to minor additional decrements in reading fluency because they were still able to read word by word. The older children, on the other hand, had the benefit of more experience with printed text, and, consequently, were developing an increased dependency on the use of peripheral retinal cues in order to enhance the rate and fluency at which a line of text

We gratefully acknowledge the assistance of Patrick Goles, principal of St. Margaret's School, Bel Air, Maryland 21014, for allowing us to enter his second- and fifth-grade classrooms. Requests for reprints should be sent to Dennis F. Fisher, Behavioral Research Directorate, Human Engineering Laboratory, Aberdeen Proving Ground, Maryland 21005. was read. Filling the spaces between the words reduced the saliency of word-boundary cues and caused the older readers to return to the less efficient word-by-word reading strategy.

To account for these effects, Hochberg has described a two-stage processing model, which includes an initial peripheral search-guidance (PSG) stage in which gross physical cues, namely word boundary and word shape, are detected in the visual periphery and prescreened for subsequent analysis. Saccadic eye movements are then initiated to bring the relevant cues and features to the fovea for higher order, cognitive search-guidance (CSG) processing. The processing sequence is cyclic, in that CSG provides PSG with global information about cues to be expected, PSG is then sensitized to the cues, and, when they are detected, eye movements are initiated to bring the cues into higher acuity regions for more in-depth analyses. It might well be argued that filling interword spaces primarily affects foveal processing, such as reducing legibility, because of factors other than word-boundary cue disruption, such as masking of initial and terminal letters (cf. Woodworth, 1938, p. 720). However, until the relative contribution of the "other factors" is more appropriately assessed, one should consider the findings of McConkie and Rayner (1975) and Spragins, Lefton, and Fisher (1976), that reduced reading speed and saccadic eye movement extent constriction, in the absence of interword spaces, implicate reduced visual peripheral processing. Fisher (1975), Fisher and Lefton (1976), Lefton and Fisher (1976), and Spragins, Lefton, and Fisher (1976) have described in more detail the activity of these processes 
and the increased repetoire in accomplished readers that allows a "falling back" to the more elemental word-by-word strategy when the task warrants it.

A contradiction is evidenced between Hochberg's interpretation and the Hochberg et al. (Note 1) data. If we are to accept the notion that experience leads to a greater dependency upon the periphery, then it should follow that, as the peripheral cue of word boundary is mutilated, older fast-reading children should show greater decrements in reading speed, and hence efficiency; however, this was not the case. In addition, to be consistent, the older slow-reading group should have mimicked the younger readers. That is, older slow readers may well lack the effective utilization of peripheral cues hypothesized to characterize the younger readers, and, therefore, little or no decrement in performance should have resulted; this was also not the case. Even a slight enhancement of peripheral cue sensitivity could account for differences found between fast and slow younger readers. It is felt that one way to resolve the interpretation to the data would be to have all subjects read paragraphs equated to their grade level, rather than to have all subjects read second-grade paragraphs.

The experiments described in the present paper are primarily concerned with the ways in which PSG and CSG might interact as beginning readers read texts with spatial and contextual variations. ${ }^{1}$ Experiment 1 examined reading speed changes occurring as second and fifth graders read their own grade-level paragraphs, when word boundaries were mutilated. Experiment 2 examined reading speed changes that occurred when fifth graders read second-grade paragraphs and vice versa. Of particular interest was the possible facilitatory effect of allowing fifth graders to read second-grade paragraphs, as was the case in the Hochberg et al. (Note 1) experiment. It was hypothesized that the interactive nature of developmental level, spatial cues (e.g., word boundary), and contextual load causes increases or reductions in reading speed and efficiency, depending upon the degree to which each is present in the task.

\section{GENERAL METHOD}

\section{Subjects}

Subjects were 22 second graders and 22 fifth graders at St. Margaret's School, Bel Air, Maryland. Eleven children from each grade level were randomly selected to participate in one of two experiments to be described. Mean ages for second and fifth graders were 8 years, 6 months, and 11 years, 6 months, respectively. All of the children were reading at or above reading grade level, as assessed by their reading teachers and Ginn 360 . Parents of the children had given prior approval for their participation in the experiment.

\section{Stimuli and Design}

The stimuli were paragraphs published by the Educational Development Laboratories, Huntington, New York (No. 376002). The paragraphs were typed with an IBM Selectric typewriter
(Artisan 12 type). The paragraphs were standardized within each grade level for difficulty, and, as a consequence, varied between grade level in length. The second-grade paragraphs averaged 63 words in length, while the fifth-grade paragraphs averaged 117 words in length. Four different paragraphs were used at each of the grade levels. Subjects in each experiment read four paragraphs plus a control or practice paragraph, which was the first paragraph found in Milne's Winnie the Pooh.

\section{EXPERIMENT 1}

\section{Procedure}

Subjects were tested individually and presented with booklets containing five paragraphs: a practice paragraph and four test paragraphs. The practice and two other paragraphs were of normal spacing (one space separating each word) and two were of filled spacing (the spaces between words were filled with a plus sign).

All subjects, whether from second or fifth grade, read paragraphs corresponding to their reading grade level. All subjects read four different paragraphs and experienced the normal and filled spacing conditions in random orders. All paragraphs appeared equally often under the two typing conditions and orders of presentation. Reading times were measured with a hand-held stopwatch and were recorded to the nearest $.10 \mathrm{sec}$. All practice and test paragraphs were read aloud to the experimenter. The subjects were instructed to read the paragraphs as quickly and as accurately as they could, and were informed that they were being timed. Those subjects requiring additional familiarization with the procedure were given such familiarization as necessary.

\section{Results and Discussion}

The response measure of time to read was converted into reading rate in words per minute (wpm) to allow for comparisons between grades, because of the two differing paragraph lengths. The reading-rate data were subjected to an analysis of variance, with groups (second vs fifth grades) as a between effect, and spacing (normal vs filled paragraphs) as a within effect. As anticipated, the main effect of grade level proved highly significant $[\mathrm{F}(1,20)=19.21, \quad \mathrm{MSe}=1,271.8, \quad \mathrm{p}<.001], \quad$ as did the main effect for spacing $[\mathrm{F}(1,20)=141.1$, $\mathrm{MSe}=152.5, \mathrm{p}<.001]$. Fifth graders read faster (141.1 wpm) than second graders (93.9 wpm), and normal type (139.6 wpm) was read faster than filled type (95.4 wpm). In addition, subjects read the practice paragraph differentially, as a function of reading grade level, with the second graders averaging $78.4 \mathrm{wpm}$, while the fifth graders read the same paragraph at the rate of $168.7 \mathrm{wpm}$.

Quite unexpectedly, the interaction of Groups by Spacing failed to reach an acceptable level of statistical significance. The finding that both groups were equally disrupted by the filled-space condition represents the first failure of the present authors' laboratory to establish the developmental involvement of the visual periphery, and the violation of a very basic assumption of the PSG. Experiment 2 reexamined word-boundary effects with the addition of contextual variations for the two age groups. 


\section{EXPERIMENT 2}

\section{Procedure}

Subjects were again tested individually. In order to assess the effects of growing contextual awareness with experience, as well as possible complementary effects due to typographical manipulations, the 11 second and 11 fifth graders used in this experiment were provided with booklets which contained two second-grade paragraphs and two fifth-grade paragraphs. One of the four paragraphs was a normally typed fifth-grade paragraph (N5); another was a normally typed second-grade paragraph (N2). Likewise, one filled-space paragraph was of a fifth-grade level (F5), and the other of a second-grade level $(\mathrm{F} 2)$. Oral reading times were again measured manually by a stopwatch to the nearest $.10 \mathrm{sec}$.

\section{Results and Discussion}

The number of words per minute was again computed for each subject for each experimental condition, in order to provide a measure of control for paragraphs of different length. The reading-rate scores were again entered into an analysis of variance, with groups (second and fifth graders) as a between effect, and reading level of the paragraphs (second and fifth) and spacing conditions (normal and filled) as within effects.

All main effects were found to be highly significant at the .001 level. Reading speed was once again found to be highly influenced by grade level $[F(1,20)=123.4$, $\mathrm{MSe}=1,629.3 \mathrm{]}$, and, once again, normal paragraphs were read significantly faster than filled paragraphs $[\mathrm{F}(1,20)=73.5, \quad \mathrm{MSe}=379.4] . \quad$ For both groups, the second-grade reading level paragraphs were read faster than fifth-grade paragraphs $[\mathrm{F}(1,20)=104.7$, $\mathrm{MSe}=258.9]$. Reading speeds for the control paragraphs were $80 \mathrm{wpm}$ and $185 \mathrm{wpm}$ for the second and fifth graders, respectively. In addition, the three-way interaction of Groups by Space Condition by Reading Grade Level proved highly significant $[F(1,20)=8.57$, $\mathrm{MSe}=279.4, \mathrm{p}<.01]$. These data are shown in Table 1 .

The reading-rate data in dicate that the $\mathrm{N} 2$ paragraphs were read fastest by both groups, and that, when both groups were confronted with F2 and N5 paragraphs, reading progressed at a somewhat slower, but equivalent rate. When fifth graders were confronted with F5 paragraphs, the reading rate was reduced to a speed which mimicked very closely that of second-grade readers reading N2 paragraphs. A smaller reduction was experienced when the second graders read the more typographically complex F5 paragraphs, compared to the $\mathrm{N} 5$ paragraphs.

The very nature of the distributions of the reading speeds found in this interaction seem to preclude

Table 1

Reading Rate by Condition in Words Per Minute

\begin{tabular}{ccrrr} 
& \multicolumn{4}{c}{ Reading Level } \\
\cline { 2 - 5 } Grade & $(\mathrm{N} 2)$ & $(\mathrm{F} 2)$ & $(\mathrm{N} 5)$ & $(\mathrm{F5})$ \\
\hline 2 & 101.3 & 58.0 & 58.6 & 42.1 \\
5 & 197.9 & 164.2 & 164.2 & 115.8 \\
\hline
\end{tabular}

the necessity for an analysis of the simple effects. A Newman-Keuls test was administered a posteriori to these data, with 2 and 20 degrees of freedom. Significant differences were found for reading rates of the fifth graders as follows: N2 paragraphs were read faster than N5 and F2 paragraphs; F2 and N5 paragraphs were read faster than F5 paragraphs (both ps $<.01$ ). The significant effects for the second-grade reading rates were as follows: N2 paragraphs were read faster than N5 and F2 paragraphs $(\mathrm{p}<.01)$; and N5 and F2 paragraphs were read faster than F5 paragraphs $(p<.05)$. One additional comparison confirmed the fact that fifth-grade readers read F5 paragraphs at the same rate as second-grade readers read $\mathrm{N} 2$ paragraphs $(\mathrm{t}<1.0)$. In essence, the simple effects seem to provide evidence for a developmental processing hierarchy and tradeoff between contextual and spatial information during reading, similar to those described in comparisons between reading and search (Fisher, 1975; Fisher \& Lefton, 1976; Lefton \& Fisher, 1976).

Establishing developmental hierarchies in beginning reading is not new (e.g., Gibson's, 1971, phonological, graphological, semantic, and syntactic feature hierarchy), but tagging them in actual reading tasks is. The functional aspects of such a hierarchy might be described as follows: The fifth-grade readers found the second-grade normally typed paragraphs easiest to read. In this case, the comprehension component was below that of normal grade level and, therefore, it is hypothesized that the peripheral retinal processes were free to operate at near the maximal efficiency possible at this grade level. It is also at this point where the confound in the Hochberg et al. (Note 1) data becomes apparent. In that experiment, all subjects read second-grade materials, and this had the consequent effect of reducing the differences between the normal- and filled-paragraph conditions and, hence, minimized the measurable effects of reduced peripheral involvement. In the present experiment, significant reductions in reading speed occurred when the text became more difficult and when reading level stayed the same, but the spatial component was degraded. The data analyses confirm that both manipulations had the same effect on functional perceptual span: that of inducing a moderate "tunnel-vision effect." As fifth graders read the easiest (N2) to the most difficult (F5) paragraphs, the greatest speed reductions occurred. It is most likely that attending to letter and word features resulted in a further reduction in functional visual field of view, as might be expected to take place when automaticity fails. In this case, fifth-grade readers were reading on a second-grade level, a finding which supports previous findings (Fisher \& Lefton, 1976; Spragins et al., 1976).

Subsequent levels of the hierarchy for second-grade readers reflect similar trends to those of the fifth-grade readers, except that the magnitude of the differences between stages is smaller, probably resulting from fluctuations in attention, even within a word-by-word 
processing strategy. It is of importance to note that any provisions for developmental differences in peripheral retinal processing must take into account the data found in the present experiments, evidencing similar reductions in reading speeds within grades, due to spatial and contextual manipulations. Therefore, the primary locus of any hypothesized reduced peripheral processing lies in the overall performance differences between grades. If a more stringent spatial manipulation were made, for example, eliminating all spaces (Fisher, 1975; Fisher \& Lefton, 1976), more dramatic reductions in reading speed of the fifth graders would be expected. Therefore, although a discrepancy between the data and the interpretation of Hochberg et al. (Note 1) has been found, it is the interpretation that must be supported in spite of those data.

\section{GENERAL DISCUSSION: A THEORETICAL EXTENTION TO PSG-CSG}

Recently, Fisher (in press) has described the complementary aspects of Hochberg's (1970) PSG-CSG notions with those of LaBerge and Samuels (1974). Although both represent highly abstract conceptualizations of the reading process, from which it is difficult to derive clear predictions for particular situations, it seems worthwhile, nonetheless, to try to take the "best" aspects of both models to arrive at a closer approximation of a description of early, accomplished, and disabled readers.

LaBerge and Samuels (1974) have described a model of automatic information processing during reading. The model involves the sequencing of visual information to phonological and episodic memories, and eventually to comprehension in the semantic memory system. Automaticity occurs when reading progresses efficiently through the text, without the necessity of directing selective attention to discriminate features of letters, words, and so forth. Automaticity fails when attention must be selectively directed to specific features of letters and words in order to attain the degree of accuracy needed for identification and/or recognition. There are three major components of developing automaticity. The first of these is feature discriminability. When new words are introduced or the features of already known words are mutilated so that discriminability is reduced, automaticity breaks down. The second is contextual level. When more difficult text is read, it is generally read at a slower rate, presumably due to the increased contextual load above that inherent in normal reading level materials. The third and unifying component is experience, or development. With experience, readers become increasingly more aware of letter feature equivalence, orthographic regularity, and contextual variation, and hence time spent on letter and word recognition is reduced. It is felt that the tradeoff between processing strategies described in the present experiments provides evidence for the presence of a mechanism regulating peripheral to cognitive searchguidance within the automaticity framework proposed by LaBerge and Samuels (1974).

The primary proposed activity of such a mechanism is that of accommodating for a processing load occurring with changes in spatial and contextual aspects of the reading material. This is accomplished by regulating the amount of information taken in during a fixation, by changing the size of the functional perceptual span. The size of the span changes in two ways: with development and increased density or information load (Lefton \& Fisher, 1976). The development of contextual and spatial sensitivities during reading acquisition is seen as critical to the efficient complementary functioning of PSG to CSG within the automaticity framework. The increasing awareness allows for the progression from elemental letter-by-letter or word-byword analyses to a more expanded informationextraction sequence, which may involve groups of words or phrases.

Adult accomplished readers sample relatively large portions of text, that is, 14 to 18 letter spaces or more to the right of fixation, at very rapid rates of $250 \mathrm{wpm}$ or more. When they were asked to search for a target without attending to the meaning of the paragraphs, their rate increased threefold (Fisher, 1975; Fisher \& Lefton, 1976). On the other hand, when given more difficult material (Tinker, 1951), or typographically mutilated paragraphs, their reading rate decreased by up to two-thirds (Fisher, 1975; Fisher \& Lefton, 1976; Spragins et al., 1976). The adult accomplished reader comes closer to representing the ideal in automatic processing with peripheral prescreening and cognitive processes in operation. When given varying contextual or typographical manipulations, the reader must attend selectively and more precisely to the lines and squiggles of the print, consequences of which are reductions in perceptual span and reading speed.

Early reading children have neither achieved automaticity nor the flexibility of adults to "fall back" on more elemental strategies; they are operating solely at the elemental strategy level. Young readers are characterized by basic slow reading speed and small perceptual spans (Spragins et al., 1976; Taylor, 1965) with very little leeway to slow down further, as they seem to be progressing through text on a nearly word-by-word basis. Little, if any, prescreening from the periphery occurs. Letter and word features and identities are scrutinized, the outcome of which ultimately culminates at some degree of anguished comprehension. Some flexibility does occur, as some facilitation in speed is found when children are asked to search rather than read, or when they are asked to read still simpler textual material, as indicated in the present experiment. Older children represent an increase in the sensitivity to the use of the periphery (Fisher \& Lefton, 1976), and, while they have still not achieved automaticity, they are showing an increased awareness in contextual and 
typographical manipulations. At successive levels of development, there is witnessed an increasing dependency on the periphery, with a peak found in the adult accomplished reader. The accomplished reader, however, still maintains the early processing strategies within his repertoire-an essential element in the peripheral to cognitive search-guidance complement to automaticity.

In short, an attempt was made at interpreting the data from the present experiments as providing support to the notion of a peripheral preprocessing to cognitive search-guidance mechanisms which are considered vital components in the acquisition of automaticity during reading. Evidence for, and the activity of, a mechanism regulating peripheral to cognitive search guidance within the automaticity framework was described. The identity of such a unifying mechanism remains obscure, partly because of the high degree of abstractness present in both theories, partly because of a lack of a more perfect "test," and partly because neither theory is complete. Hochberg's (1970) notions include peripheral prescreening and eye movements in the cueacquisition sequence, without specifying the detailed higher order processing lumped into cognitive search guidance. LaBerge and Samuels (1974), on the other hand, specify information processing in feature acquisition, extraction, and consolidation, whereby features are integrated into phonological, episodic, and semantic memories, but omit contributions to automaticity stemming from peripheral prescreening and eye movements. Both depend heavily upon development or experience as a means of effecting efficient reading strategy, while remaining elusive about the evolution of comprehensibility. Taken together as complements, these notions provide a more complete, highly definitive analysis of the progression of skill acquisition that is developmentally dependent and sensitive to contextual and typographical constraints, but there is still much to be done.

\section{REFERENCE NOTE}

1. Hochberg, J., Levin, H., \& Frail, C. Studies of oral reading: VII. How interword spaces affect reading. Unpublished report, Cornell University, 1966.

\section{REFERENCES}

FISHER, D. F. Reading and visual search. Memory \& Cognition, 1975, 3, 188-196.

Fisher, D. F. Dysfunction in reading disability: There's more than meets the eye. In L. Resnick \& P. Weaver (Eds.), Theory and practice of early reading (Vol. 1). Lawrence Erlbaum, Hillsdale, N.J: in press.

Fisher, D. F., \& Lefton, L. A. Peripheral information extraction: A developmental examination. Journal of Experimental Child Psychology, 1976, 21, 77-93.

Gibson, E. J. Perceptual learning and the theory of word perception. Cognitive Psychology, 1971, 2, 351-368.

HoCHBERG, J. Components of literacy: Speculation and exploratory research. In H. Levin \& J. P. Williams (Eds.), Basic studies on reading. New York: Basic Books, 1970. Pp. 74-89.

LaBerge, D., \& SAmuels, S. J. Toward a theory of automatic information processing in reading. Cognitive Psychology, 1974, 6, 293-323.

Lefton, L. A., \& Fisher, D. F. Information extraction during visual search: A developmental progression. Journal of Experimental Child Psychology, 1976, 22, 346-361.

MCCONKIE, G. W., \& RAYNER, K. The span of the effective stimulus during a fixation in reading. Perception \& Psychophysics, 1975, 17, 578-586.

Milne, A. A. Winnie the pooh. New York: Dell, 1954.

Spragins, A. B., Lefton, L. A., \& Fisher, D. F. Eye movements while reading and searching spatially transformed text: A developmental examination. Memory \& Cognition, 1976, 4, 36-42.

TAYLOR, S. E. Eye movements in reading: Facts and fallacies. American Educational Research Journal, $1965,2,187-202$.

Tinker. M. A. Fixation pauses duration in reading. Journal of Educational Research, 1951, 44, 471-479.

Woodworth, R. S. Experimental psychology. New York: Holt, 1938.

\section{NOTE}

1. We are presently engaged in a series of experiments designed to examine peripheral retinal processes in disabled readers, as compared to slow and normal readers. Until those determinants on quality of reading can be made, we have decided to omit such group comparisons from the present experiments.

(Received for publication August 16, 1976; revision received November 23,1976 .) 